\title{
Adjuvant Docetaxel and Abbreviated Androgen Deprivation Therapy in Patients with High Risk Prostate Cancer
}

\author{
Jose G. Bazan, Christopher R. King, James D. Brooks and Sandy Srinivas*
}

Oncology 875 Blake Wilbur Drive, Stanford, CA 94305 USA

Summary: Objectives: To evaluate the safety and efficacy of docetaxel and an abbreviated course of hormonal therapy in patients with high risk prostate cancer after radical prostatectomy (RP) or radiation therapy (RT).

Materials and Methods: A total of 21 patients were enrolled between June 2004 and October 2005. Nine patients were treated with RP and eleven patients with RT. High risk of disease recurrence was defined by at least one of the following: node positive disease post-operatively, capsule involvement, seminal vesicle involvement, Gleason score $\geq 8,>50 \%$ of core biopsies that are positive, clinical stage T2c or T3, or pre-operative PSA > 15 plus Gleason score of 7. Treatment consisted of 3 cycles of docetaxel chemotherapy and hormonal therapy consisted of an LHRH analog (Lupron) with or without anti-androgen therapy (Casodex) for six months.

Results: Overall, treatment was well tolerated with minimal toxicity. One patient experienced grade 4 hyperglycemia and one patient experienced grade 3 transaminitis. At a median follow-up of 47.5 months, 7 patients (35\%) have progressed. Four-year estimated Kaplan-Meier biochemical progression free survival (KM-bPFS) was $63.7 \%$.

Conclusions: Docetaxel and an abbreviated course of hormonal therapy after RP or RT in patients with high risk prostate cancer is feasible and safe. The ongoing randomized phase III trial of abbreviated 6 months hormonal therapy with radiation therapy with or without docetaxel in patients with high risk prostate cancer will elucidate the role of docetaxel on biochemical progression-free survival and overall survival in this group of high risk patients with prostate cancer.

Keywords: Abbreviated androgen deprivation; high risk; prostate cancer; radiation; radical prostatectomy; docetaxel chemotherapy.

\section{INTRODUCTION}

Several criteria have been identified that deem patients to be at high risk for recurrence following definitive treatment for prostate cancer. They include Gleason score > 7, preoperative PSA > $20 \mathrm{ng} / \mathrm{ml}$, pathologic $\mathrm{T}$ stage $>\mathrm{T} 2 \mathrm{~b}$, and postoperatively, involvement of nodes, capsule, and seminal vesicles. [1]

Few high-risk patients are cured with radiotherapy or prostatectomy alone, although some high risk patients benefit from the addition of neoadjuvant and adjuvant androgen deprivation in addition to radiotherapy, both in terms of local control and overall survival. Currently, there is no standard adjuvant or neoadjuvant therapy for high-risk RP patients. Neoadjuvant hormonal therapy (NHT) has not been shown to improve biochemical recurrence rates in RP patients [2]. Addition of androgen deprivation therapy to patients post prostatectomy with microscopic nodal metastases resulted in improved survival [3]. In RT patients, the addition of long term androgen ablation offers significantly better disease-specific survival and overall survival rates compared with radiotherapy alone [4-8]. Nonetheless, some patients continue to have a high risk of local and subsequent distant

*Address correspondence to this author at the Oncology 875 Blake Wilbur Drive Stanford, CA 94305 USA; Tel: (650)725-2078; Fax: (650)736-1640;

E-mail: sandysri@stanford.edu failure, despite the best combination treatment. Few studies have examined the use of docetaxel as adjuvant therapy with or without hormonal therapy in high risk patients treated definitively with RT.

Adjuvant therapy with vinblastine, doxorubicin and mitomycin has been used in patients with high risk disease along with external radiation and has shown to have a superior outcome [9]. In addition, a randomized trial of 96 patients was conducted using adjuvant flutamide in combination with mitoxantrone versus flutamide alone. This study demonstrated a survival advantage to the chemotherapy group [10]. SWOG 9921, a randomized trial of 2 years of ADT with or without mitoxantrone post prostatectomy closed early after increase incidence of leukemia in the mitoxantrone arm [11]. Adjuvant trials with more contemporary drugs are clearly needed.

The most active regimens in hormone refractory prostate cancer have been those containing the vinca alkaloids, anthracyclines and the taxanes. Two randomized trials have established that the semi-synthetic taxane docetaxel, in combination with estramustine or prednisone, improves both overall survival and PSA response rates (defined as a $50 \%$ decrease in PSA) when compared to mitoxantrone-based chemotherapy in patients with hormone refractory prostate cancer $[12,13]$. 
Docetaxel administered on a weekly schedule has activity in a variety of tumor types including breast, lung, and prostate cancer. Weekly dosing minimizes the hematologic toxicity and provides a dose-intense strategy for administration. Docetaxel has been studied as a weekly single-agent regimen at a dose of $36 \mathrm{mg} / \mathrm{m}^{2}$ for 6 out of 8 weeks, followed by a two-week rest period in the treatment of hormone-refractory prostate cancer. The PSA response rate was $41 \%$ and $46 \%$ in two recent trials incorporating this regimen $[14,15]$. The therapy was well tolerated with grade 3 and 4 hematologic toxicity occurring in less than $25 \%$ of patients.

Because of the efficacy and tolerability of weekly docetaxel, we chose to combine it with LHRH analogs for the adjuvant treatment of high risk prostate cancer. Long term androgen ablation therapy in the treatment of prostate cancer has been associated with significant adverse effects that impact both overall health and quality of life [16], and it is therefore reasonable to consider a shorter duration of hormonal therapy. The recommended dose of weekly docetaxel is $35-40 \mathrm{mg} / \mathrm{m}^{2} /$ week for 6 out of 8 weeks. The same dose intensity can be achieved on a 3 out of 4 week basis and this schedule may be better tolerated.

We report biochemical progression-free survival (bPFS) and toxicity of docetaxel and an abbreviated course of hormonal therapy in a post RP or RT group of patients with high risk prostate cancer in a phase II clinical trial.

\section{MATERIALS AND METHODS}

\section{Study Design}

This is a phase II trial evaluating the toxicity and efficacy of adjuvant docetaxel and hormonal therapy after RP or RT in patients with high-risk prostate cancer. We conducted this study under the review and approval of the Stanford institutional review board. Between June 2004 and October 2005, 21 patients were prospectively enrolled at the Stanford University Medical Center and participating hospitals.

\section{Patient Population}

Patients were identified prospectively at participating institutions. One withdrew consent and 20 are evaluable for response and toxicity. Nine patients underwent RP and eleven patients underwent RT for biopsy-proven prostate cancer. To be eligible, patients that underwent radical prostatectomy were required to have undetectable PSA postoperatively (PSA $<0.02 \mathrm{ng} / \mathrm{ml}$ ). Patients were required to have high-risk disease as defined by at least one of the following: node positive disease post-operatively, capsule involvement, seminal vesicle involvement, Gleason score $\geq 8$, $>50 \%$ of core biopsies that are positive, clinical stage T2c or T3, or pre-operative PSA > 15 plus Gleason score of 7. Additional inclusion criteria included ECOG performance status $0-1$, no contraindication to Taxotere or other drugs formulated with polysorbate 50 , no baseline peripheral neuropathy $>$ grade 1 as defined by the Common Terminology Criteria for Adverse Events (CTCAE) version 3.0, and no other malignancy for 5 years or more except for nonmelanomatous skin cancer.

\section{Treatment}

Hormonal therapy consisted of LHRH analog (Luprolide) with or without anti-androgen therapy (Casodex). All patients received intramuscular LHRH analog for six months. For patients that underwent RP, LHRH analog injections began within three to four weeks following surgery. Those patients that received radiation as their primary therapy started LHRH analog two months prior to day 1 of radiation. All patients continued hormonal therapy for a total of six months.

Chemotherapy began within 10-12 weeks after completion of surgery or radiation in all patients. Treatment consisted of 3 cycles of $35 \mathrm{mg} / \mathrm{m}^{2}$ docetaxel administered intravenously over 30 minutes on days 1,8 , and 15 of each 28 day cycle. Prior to each infusion, patients were premedicated with $12 \mathrm{mg}$ dexamathesone orally $(4 \mathrm{mg}$ given the night before, the morning of, and the evening after docetaxel administration.

Pretreatment evaluation consisted of physical examination, complete blood count, routine chemical profile, PSA and bone scan. Patients were re-evaluated weekly during treatment and then every 4 months following treatment with physical examination, complete blood count, routine chemical profile, and PSA.

\section{Sample Size Justification}

The primary endpoint of this study was 2-year bPFS. The 2 yr bPFS was chosen as an endpoint based on our assumption that hormonal recovery would be achieved in this time period. Accrual of 21 patients was deemed sufficient to detect an absolute $25 \%$ difference in the two-year PFS rate between the experimental treatment and historical controls with a 2 -sided 0.05 significance level and $80 \%$ power.

\section{Outcomes}

We used the Kaplan-Meier method to determine bPFS. Log-rank analysis was used for differences in proportions. Biochemical PFS was defined as the time from initiation of chemotherapy to progression. In patients who have undergone RP, progression was defined by PSA $0.02 \mathrm{ng} / \mathrm{ml}$ or greater. For patients who have undergone radiation therapy, progression was defined by the American Society of Therapeutic Radiology and Oncology (ASTRO) guidelines of three consecutive rises in serum PSA [17].

Adverse events were assessed rigorously during treatment and at subsequent follow-ups using patient history and laboratory assessments, where appropriate. All toxicities were graded according to the National Cancer Institute Common Terminology Criteria for Adverse Events, version $3.0[18]$.

All statistical analysis was performed using SAS v.9.2.

\section{RESULTS}

\section{Patient Characteristics}

Baseline characteristics of the twenty patients with high risk prostate cancer treated with RP or RT are shown in Table 1. Thirteen patients had combined androgen blockade with both Luprolide and bicalutamide for 6 months and 7 had luprolide alone. Mean pretreatment PSA was significantly higher in the RT group $(25.9 \mathrm{ng} / \mathrm{ml}$ vs. $12.9 \mathrm{ng} / \mathrm{ml}, p=$ 0.02 ). Patients in the radiotherapy group tended to have a higher Gleason score, with $73 \%$ having a Gleason score $\geq 8$ 
Table 1. Baseline Patient Characteristics

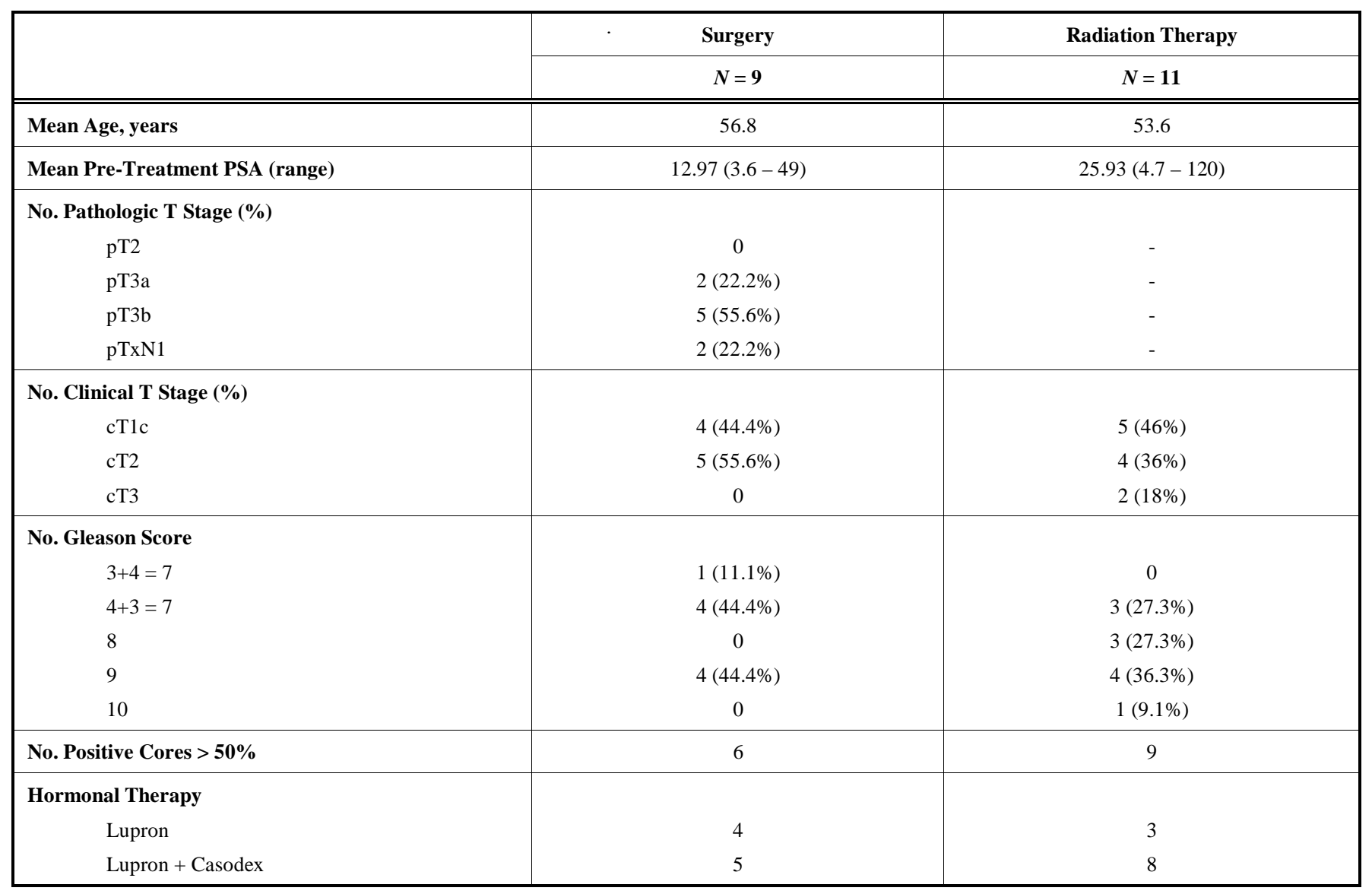

compared to $44 \%$ in the RP group, although this did not reach statistical significance $(p=0.2)$. In the RP group, pathologic findings included two patients $(22 \%)$ with nodepositive disease. All RP patients had pT3 disease.

\section{Toxicity}

A total of 178 out of the planned 180 doses of taxotere were delivered. Two doses $(1.1 \%)$ were omitted, and there were no dose reductions. The omitted doses were related to toxicity in one patient and to a viral illness in another patient.

Overall, the treatment with taxotere was well-tolerated. No grade 3 or higher hematologic toxicities were observed. One patient experienced grade 4 hyperglycemia, most likely related to dexamethasone. The patient responded to an insulin regimen and a dose reduction of dexamethasone. One patient experienced a grade 3 transaminitis. This resolved without dose reduction. No other grade 3 or higher toxicities were observed. The most common toxicities are listed in Table 2.

\section{PFS Analysis}

Twenty patients were evaluable for outcomes analysis, and all patients were alive at the time of this analysis. At a median follow-up of 47.5 months, 7 patients $(35 \%)$ had progressed. Four-year bPFS was $63.7 \%$ (Fig. 1). The overall median time to progression was 16 months (range, $9-21$ months). Median PFS for the cohort was not reached, al- though the lower bound of the $95 \%$ confidence limit is estimated at 17 months.

Table 2. Acute Toxicity Data

\begin{tabular}{|l|l|}
\hline NCI Toxicity Definition & Toxicity Grade \\
\hline \hline Fatigue & Grade 1 - 2 \\
\hline Diarrhea & $19(95 \%)$ \\
\hline Nail Changes & $8(40 \%)$ \\
\hline Dysguesia & $5(20 \%)$ \\
\hline Neuropathy & $7(35 \%)$ \\
\hline Anemia & $4(20 \%)$ \\
\hline Leukopenia & $8(40 \%)$ \\
\hline Transaminitis & $2(10 \%)$ \\
\hline & $5(20 \%)$ \\
\hline Transaminitis & Grade 3 \\
\hline & $1(5 \%)$ \\
\hline Hyperglycemia & Grade 4 \\
\hline
\end{tabular}




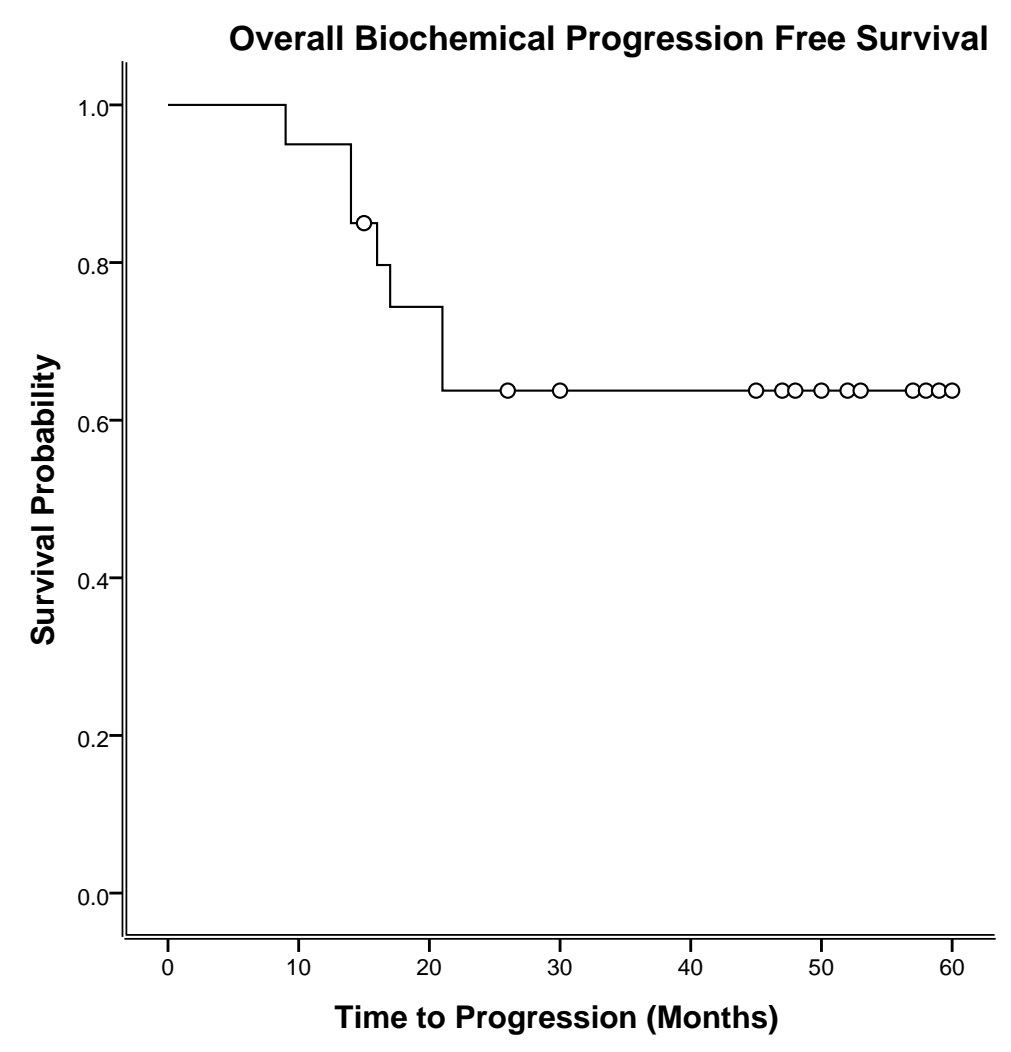

Fig. (1). Overall biochemical progression free survival.

Biochemical PFS was significantly higher for patients treated with radiation therapy compared to those treated with RP. One patient in the radiation therapy group progressed whereas six patients in the RP group progressed. Four year bPFS was $91 \%$ in the RT group and $26 \%$ in the RP group. Median bPFS in the RP group was 21 months (95\% Confidence Interval: 17 - 25 months).

Data on testosterone recovery was available in 8 out of the 10 patients in the RP group that did no progress. All eight patients achieved normalization of testosterone levels (> $200 \mathrm{ng} / \mathrm{dL}$ ). The median testosterone level at the time of recovery was $327.5 \mathrm{ng} / \mathrm{dL}$ (range, 214-394 $\mathrm{ng} / \mathrm{dL}$ ), and the median time to testosterone recovery after completion of therapy was 17 months (range, 4- 30 months).

\section{DISCUSSION}

In this phase 2 study, adjuvant weekly docetaxel along with hormonal therapy was given to patients with a high risk of recurrence of prostate cancer based on clinical and/or pathologic characteristics. The primary treatment for all patients was either RP or RT. Patients that underwent RP received hormonal therapy in the adjuvant setting whereas patients that underwent RT received neoadjuvant hormonal therapy (NHT) and adjuvant hormonal therapy (AHT). Treatment was well-tolerated, and the overall 2-year bPFS rate was $61 \%$. The benefit of adjuvant docetaxel with hormonal therapy was mostly confined to the patients that received $\mathrm{RT}$ as definitive therapy.

In this study, chemotherapy with docetaxel was given on a weekly basis. Overall, toxicity was mild, and all toxicities were reversible. One patient experienced a grade 3 transaminitis ( $>5 \mathrm{X} \mathrm{ULN}$ ) that resolved without dose modification or intervention. One other patient experienced grade 4 $(>500 \mathrm{mg} / \mathrm{dl})$ hyperglycemia, most likely related to dexamethasone. All other toxicities were grade 1 or 2 and well tolerated.

Docetaxel has been studied in the adjuvant setting in patients with high risk prostate cancer after RP. Kibel recently showed that $60.5 \%$ of the patients progressed at a median follow-up time of 29 months [19]. Similarly, in our study, $74 \%$ of the patients treated with RP progressed. However, only $9 \%$ of patients treated definitively with RT progressed on this trial. No studies have examined the use of docetaxel as adjuvant therapy with or without hormonal therapy in high risk patients treated definitively with RT.

The optimal treatment for patients that undergo definitive therapy and that have high risk pathologic features has not yet been determined. All of the surgical patients in this study had locally confined disease by clinical criteria and were subsequently found to have high risk features based on pathology. Recently, several randomized studies have shown that adjuvant RT after RP in patients with high risk pathologic characteristics improves biochemical progression-free survival and local control, but not distant-metastasis free survival or overall survival $[20,21]$. RTOG 9902, a randomized trial evaluating the combination chemotherapy with paclitaxel, estramustine and etoposide was closed early due to excess thrombo embolic disease [22].

In our study, the rate of progression at four years in the RP patients that received adjuvant chemotherapy and hormonal therapy was high, suggesting the need for more novel approaches for this group of high risk patients. This trial was not designed to compare radiation versus RP; however, pa- 
tients with RT with an abbreviated course of ADT did have a good PFS. The inferior results in the surgical group could be from the small sample size or the need for post operative radiation following RP. The only pathologic tissue in the patients who underwent radiation was the biopsy pre therapy and that group may have had fewer node positive patients explaining the difference.

Only one patient that received RT as definitive therapy relapsed in this study. The standard of care for high-risk prostate cancer is the combination of long-term hormonal therapy with radiation therapy based on four randomized studies and a metaanalysis [4, 6-8, 23]. Six months of hormonal therapy with radiation was inferior compared to 3 years of ADT [24] Numerous randomized studies have shown that the combination of NHT and RT improves either overall survival or biochemical relapse-free survival in patients with locally advanced prostate cancer [5, 7, 25, 26]. In this study, the goal of adding adjuvant docetaxel was to attenuate the duration of hormonal therapy in an effort to minimize the adverse effects associated with long-term androgen ablation.

While the six months of hormonal therapy in the RP group may not have been adequate enough to achieve local control, it is more likely that there is a biological interaction between hormonal therapy and radiation therapy thereby accounting for the improved bPFS in the RT patients compared to the RP patients. It has been hypothesized that the mechanism underlying the benefit of NHT may be due to a T-cell mediated response [27]. According to Mercader, androgen ablation with hormonal therapy leads to T-cell infiltration of the prostate that peaks 3 - 4 weeks into treatment. This infiltration results in increased apoptosis in the prostate and possibly within regional lymph nodes thereby enhancing the efficacy of radiation therapy. Current research is further looking into the possible interaction between the timing of hormonal therapy and radiation field size (whole pelvic vs. prostate only), which has been found upon further analysis of RTOG 94-13 [28].

Limitations of our study include the small sample size. Some of the more serious adverse effects of weekly docetaxel may have been missed due to the sample size. In addition, at the time of the design of this trial, a regimen of weekly docetaxel was chosen as opposed to docetaxel every three weeks under the assumption that weekly docetaxel is better tolerated. Since then, Tannock published a randomized trial demonstrating that a weekly schedule of docetaxel was not associated with a lower rate of adverse events or improved outcomes [13]. As a result, docetaxel every three weeks should be used in future studies.

\section{CONCLUSIONS}

Our study provides evidence that weekly docetaxel and hormonal therapy in patients with high risk prostate cancer that have undergone RP or RT is feasible and safe. The primary efficacy endpoint of 2 -year bPFS $\geq 61 \%$ was reached. The 4-year bPFS was significantly higher in the patients treated with RT versus those treated with RP. Thus, NHT followed by RT, adjuvant chemotherapy and AHT warrants further study. The ongoing randomized phase III trial of neoadjuvant hormonal therapy followed by radiation therapy and adjuvant hormonal therapy with or without docetaxel in patients with high risk prostate cancer will elucidate the role of docetaxel on progression-free survival and overall survival in this group of high risk patients with prostate cancer.

\section{REFERENCES}

[1] Eisenberger MA. Therapeutic considerations for patients with highrisk, nonmetastatic, prostate cancer. Semin Urol Oncol 2002; 20: 19-23.

[2] Klotz LH, Goldenberg SL, Jewett MA, et al. Long-term followup of a randomized trial of 0 versus 3 months of neoadjuvant androgen ablation before radical prostatectomy. J Urol 2003; 170: 791-4.

[3] Messing EM, Manola J, Yao J, et al. Immediate versus deferred androgen deprivation treatment in patients with node-positive prostate cancer after radical prostatectomy and pelvic lymphadenectomy. Lancet Oncol 2006; 7: 472-9.

[4] Bolla M, Collette L, Blank L, et al. Long-term results with immediate androgen suppression and external irradiation in patients with locally advanced prostate cancer (an EORTC study): a phase III randomised trial. Lancet 2002; 360: 103-6.

[5] D'Amico AV, Loffredo M, Renshaw AA, et al. Six-month androgen suppression plus radiation therapy compared with radiation therapy alone for men with prostate cancer and a rapidly increasing pretreatment prostate-specific antigen level. J Clin Oncol 2006; 24: 4190-5.

[6] Efstathiou JA, Bae K, Shipley WU, et al. Cardiovascular Mortality and Duration of Androgen Deprivation for Locally Advanced Prostate Cancer: Analysis of RTOG 92-02. Eur Urol 2008; 54(4): 816-23.

[7] Pilepich MV, Winter K, John MJ, et al. Phase III radiation therapy oncology group (RTOG) trial 86-10 of androgen deprivation adjuvant to definitive radiotherapy in locally advanced carcinoma of the prostate. Int J Radiat Oncol Biol Phys 2001; 50: 1243-52.

[8] Pilepich MV, Winter K, Lawton CA, et al. Androgen suppression adjuvant to definitive radiotherapy in prostate carcinoma--longterm results of phase III RTOG 85-31. Int J Radiat Oncol Biol Phys 2005; 61: 1285-90.

[9] Bagley CM, Jr., Lane RF, Blasko JC, et al. Adjuvant chemohormonal therapy of high risk prostate carcinoma. Ten year results. Cancer 2002; 94: 2728-32.

[10] Wang J, Halford S, Rigg A, et al. Adjuvant mitozantrone chemotherapy in advanced prostate cancer. BJU Int 2000; 86: 67580 .

[11] Flaig TW, Tangen CM, Hussain MH, et al. Randomization reveals unexpected acute leukemias in Southwest Oncology Group prostate cancer trial. J Clin Oncol 2008; 26: 1532-6.

[12] Petrylak DP, Tangen CM, Hussain MH, et al. Docetaxel and estramustine compared with mitoxantrone and prednisone for advanced refractory prostate cancer. N Engl J Med 2004; 351: 1513-20.

[13] Tannock IF, de Wit R, Berry WR, et al. Docetaxel plus prednisone or mitoxantrone plus prednisone for advanced prostate cancer. $\mathrm{N}$ Engl J Med 2004; 351: 1502-12.

[14] Beer TM, Pierce WC, Lowe BA, et al. Phase II study of weekly docetaxel in symptomatic androgen-independent prostate cancer. Ann Oncol 2001; 12: 1273-9.

[15] Berry W, Dakhil S, Gregurich MA et al. Phase II trial of singleagent weekly docetaxel in hormone-refractory, symptomatic, metastatic carcinoma of the prostate. Semin Oncol 2001; 28: 8-15.

[16] Holzbeierlein JM, McLaughlin MD, Thrasher JB. Complications of androgen deprivation therapy for prostate cancer. Curr Opin Urol 2004; 14: 177-83.

[17] Consensus statement: guidelines for PSA following radiation therapy. American Society for Therapeutic Radiology and Oncology Consensus Panel. Int J Radiat Oncol Biol Phys 1997; 37: 1035-41.

[18] Trotti A, Colevas AD, Setser A, et al. CTCAE v3.0: development of a comprehensive grading system for the adverse effects of cancer treatment. Semin Radiat Oncol 2003; 13: 176-81.

[19] Kibel AS, Rosenbaum E, Kattan MW, et al. Adjuvant weekly docetaxel for patients with high risk prostate cancer after radical prostatectomy: a multi-institutional pilot study. J Urol 2007; 177: 1777-81.

[20] Bolla M, van Poppel H, Collette L, et al. Postoperative radiotherapy after radical prostatectomy: a randomised controlled trial (EORTC trial 22911). Lancet 2005; 366: 572-8. 
[21] Thompson IM Jr, Tangen CM, Paradelo J, et al. Adjuvant radiotherapy for pathologically advanced prostate cancer: a randomized clinical trial. JAMA 2006; 296: 2329-35.

[22] Rosenthal SA, Bae K, Pienta KJ, et al. Phase III multi-institutional trial of adjuvant chemotherapy with paclitaxel, estramustine, and oral etoposide combined with long-term androgen suppression therapy and radiotherapy versus long-term androgen suppression plus radiotherapy alone for high-risk prostate cancer: preliminary toxicity analysis of RTOG 99-02. Int J Radiat Oncol Biol Phys 2009; 73: 672-8

[23] Bria E, Cuppone F, Giannarelli D, et al. Does hormone treatment added to radiotherapy improve outcome in locally advanced prostate cancer?: meta-analysis of randomized trials. Cancer 2009; 115: 3446-56.

[24] Bolla M, de Reijke TM, Van Tienhoven G, et al. Duration of androgen suppression in the treatment of prostate cancer. N Engl J Med 2009; 360: 2516-27.
[25] Denham JW, Steigler A, Lamb DS, et al. Short-term androgen deprivation and radiotherapy for locally advanced prostate cancer: results from the Trans-Tasman Radiation Oncology Group 96.01 randomised controlled trial. Lancet Oncol 2005; 6: 841-50.

[26] Laverdiere J, Nabid A, De Bedoya LD, et al. The efficacy and sequencing of a short course of androgen suppression on freedom from biochemical failure when administered with radiation therapy for T2-T3 prostate cancer. J Urol 2004; 171: 1137-40.

[27] Mercader M, Bodner BK, Moser MT, et al. T cell infiltration of the prostate induced by androgen withdrawal in patients with prostate cancer. Proc Natl Acad Sci USA 2001; 98 : 14565-70.

[28] Lawton CA, DeSilvio M, Roach M, et al. An update of the phase III trial comparing whole pelvic to prostate only radiotherapy and neoadjuvant to adjuvant total androgen suppression: updated analysis of RTOG 94-13, with emphasis on unexpected hormone/ radiation interactions. Int J Radiat Oncol Biol Phys 2007; 69: 646-55.

(C) Bazan et al.; Licensee Bentham Open.

This is an open access article licensed under the terms of the Creative Commons Attribution Non-Commercial License (http://creativecommons.org/licenses/ by-nc/3.0/) which permits unrestricted, non-commercial use, distribution and reproduction in any medium, provided the work is properly cited. 